\begin{tabular}{|c|l|}
\hline Title & Validity criteria of the discrete dipole approximation \\
\hline Author(s) & $\begin{array}{l}\text { Zubko, Evgenij; Petrov, Dmitry; Grynko, Y evgen; Shkuratov, Y uriy; Okamoto, Hajime; Muinonen, Karri; Nousiainen, } \\
\text { Timo; Kimura, Hiroshi; Y amamoto, Tetsuo; Videen, Gorden }\end{array}$ \\
\hline Citation & $\begin{array}{l}\text { A pplied Optics, 49(8), 1267-1279 } \\
\text { https://doi.org/10.1364/A 0.49.001267 }\end{array}$ \\
\hline Issue Date & 2010-03-10 \\
\hline Doc URL & http://hdl.handle.net/2115/50065 \\
\hline Rights & ○ 2010 Optical Society of A merica \\
\hline Type & article \\
\hline File Information & A 049-8_1267-1279.pdf \\
\hline
\end{tabular}

Instructions for use 


\title{
Validity criteria of the discrete dipole approximation
}

\author{
Evgenij Zubko, ${ }^{1,2,3, *}$ Dmitry Petrov, ${ }^{2}$ Yevgen Grynko, ${ }^{4}$ Yuriy Shkuratov, ${ }^{2}$ \\ Hajime Okamoto, ${ }^{1}$ Karri Muinonen, ${ }^{3,5}$ Timo Nousiainen, ${ }^{6}$ \\ Hiroshi Kimura, ${ }^{7}$ Tetsuo Yamamoto, ${ }^{8}$ \\ and Gorden Videen ${ }^{9}$ \\ ${ }^{1}$ Graduate School of Science, Tohoku University, Aoba, Aramakiaza, Aoba-ku, Sendai 980-8578, Japan \\ ${ }^{2}$ Institute of Astronomy, Kharkov National University, 35 Sumskaya Street, Kharkov 61022, Ukraine \\ ${ }^{3}$ Observatory, Post Office Box 14, Fl-00014 University of Helsinki, Finland \\ ${ }^{4}$ BASF SE, Carl-Bosch-Strasse 38, 67056, Ludwigshafen, Germany \\ ${ }^{5}$ Finnish Geodetic Institute, Post Office Box 15, FI-02431 \\ Masala, Finland \\ ${ }^{6}$ Department of Physics, Post Office Box 68, FI-00014 \\ University of Helsinki, Helsinki, Finland \\ ${ }^{7}$ Center for Planetary Science, Graduate School of Science, Kobe University, \\ Nada-ku Rokkodai-cho 1-1, Kobe 657-8501, Japan \\ ${ }^{8}$ Institute of Low Temperature Science, Hokkaido University, \\ Kita-ku North 19 West 8, Sapporo 060-0819, Japan \\ ${ }^{9}$ Space Science Institute, 4750 Walnut Street, Suite 205, \\ Boulder, Colorado 80301, USA \\ ${ }^{\star}$ Corresponding author: ezubko@rambler.ru
}

Received 30 November 2009; revised 7 January 2010; accepted 15 January 2010;

posted 22 January 2010 (Doc. ID 120631); published 4 March 2010

\begin{abstract}
There are two widely accepted restrictions on the application of the discrete dipole approximation (DDA) in the study of light scattering by particles comparable to the wavelength: (1) when considering dielectric particles, the size of the cells must satisfy the condition $k d|m|<0.5$, where $k$ is the wavenumber, $d$ is the size of the cells, and $m$ is the complex refractive index of the constituent material and (2) when considering conductive particles, the size of the cells must be small enough to reproduce sufficiently the evolution of the electromagnetic field in the skin layer. We examine both restrictions when the DDA is applied to irregularly shaped particles and show that its restrictions are not as strong as is widely accepted. For instance, when studying irregularly shaped particles averaged over orientations, even at $k d|m|=1$, the DDA provides highly accurate numerical results. Moreover, we show that the impact of using large constituent cells is similar to that produced by surface roughness; therefore, the replacement of the target particle by an array of large constituent cells has the same effect, qualitatively, as incorporating additional small-scale surface roughness on the particle. Such a modification of the target particle can be desirable in many practical applications of DDA when irregularly shaped particles are considered. When applying DDA to conductive, nonspherical particles, the insufficient description of the electromagnetic field in the skin layer does not lead to a violation of the Maxwell equations, although it has a visible but nonmajor influence on the light-scattering properties of the target. (C) 2010 Optical Society of America
\end{abstract}

OCIS codes: $\quad 290.5825,290.5850,290.5855$.

0003-6935/10/081267-13\$15.00/0

(C) 2010 Optical Society of America 


\section{Introduction}

The discrete dipole approximation (DDA), which is also called the coupled-dipole method, is a powerful technique to compute light scattering by particles having sizes comparable to the wavelength. Within this approach, the particles can be of arbitrary shape and internal composition. In the DDA, the particles are mapped onto an array of small (in comparison to the wavelength) volumes that together reproduce the shape and internal optical response of the original particles. Light scattering by small volumes, or electric dipoles, is well studied, and their scattered field takes the form of a simple analytic expression. Therefore, the substitution allows for the reformulation of the initial light-scattering problem into that of light scattering by an array of coupled small cells. In this way, the difficulties accompanying an analytical consideration of light scattering by irregularly shaped particles can be exchanged for a computational routine of an iterative solution of a system of linear equations. Note that the difficulties mentioned above appear already at the stage of formulation of the problem of light scattering by an irregularly shaped particle, whereas within the DDA, it is no more difficult to consider an irregular particle. Indeed, it is necessary only to specify the optical properties of the local material corresponding to the dipole locations. As a consequence, a system of linear algebraic equations describing the light scattering is obtained without significant effort or computations.

The DDA formulation was published in 1973 [1] by Purcell and Pennypacker who considered light scattering by rectangular grains consisting of up to 150 dipoles. The size parameter of the equal-volume sphere $x_{\text {eq }}=2 \pi r / \lambda$, where $r$ is the radius and $\lambda$ is the wavelength of the incident light, was restricted to a value of 2 ; i.e., target particles were smaller than the incident wavelength. This experiment with the DDA stimulated further research. Unfortunately, in the seventies and early eighties, the number of researchers actively using the DDA was limited to those who had access to significant computer resources. Nevertheless, as early as 1975, the DDA had been used to study rectangular magnetite grains [2] and, in 1979, to inhomogeneous spheres [3].

Simultaneously, there have been steps taken to accelerate computations. The acceleration of the DDA algorithm can be achieved by decreasing the total number of iterations and speeding up the time for a single iteration. In the former case, more efficient iterative algorithms have been developed [4,5], whereas in the latter case, the fast Fourier transformation (FFT) has been implemented [6]. This latter improvement is especially significant, allowing for an increase in the number of dipoles by more than an order of magnitude.

Another important enhancement of the DDA has had no effect on the speed of calculations but rather on the accuracy of the results: it concerns the relation between the refractive index of the constituent material and the polarizability of the dipoles. Purcell and Pennypacker used the simplest ClausiusMossotti relation [1], whereas Draine incorporated the "radiative reaction" [5]. Later, Draine and Goodman proposed the so-called lattice dispersion relation, which further improved DDA accuracy [7]. At present, the latter correction is widely used and is incorporated in our implementation.

In the development of the DDA, the question about the range of applicability has been actively discussed $[1,4,5,8]$. The most important and obvious restriction is the size of discretized volumes replacing the initial dielectric target: these cells must be much smaller than the wavelength to represent the target adequately; however, there is a clear desire to make them as large as possible in order to increase the speed of calculations. The final formulation of the restriction on size of these cells has been summarized as follows [8]:

$$
k d|m|<A .
$$

Here $k=2 \pi / \lambda$ is the wavenumber, $d$ is the size of the cell, $m$ is the complex refractive index of a constituent material, and $A$ is some constant that can be found experimentally.

As was shown in [8], when $A=1$, the DDA provides quite reasonable accuracy in the cross sections of absorption and scattering, whereas the angular profile of intensity may reveal fractional errors exceeding $30 \%$. Draine and Flatau recommend using the criterion expressed in Eq. (1) at $A=0.5$ when accurate calculations of the intensity profile are desired [9]. The authors imply that reducing $A$ in Eq. (1) can be done, as necessary, to describe the target shape satisfactorily $[\underline{5}, \underline{8}]$. However, this validity criterion has not undergone significant revision since 1994 (see, for example, recent benchmark studies of the DDA $[10,11])$.

Another restriction of the DDA concerns the case of a target composed of conductive material. When applying the DDA to such a particle, we have to be sure that the cells forming a target particle remain electric dipoles, i.e., that the relative contribution of the magnetic dipole moment to the light scattering is sufficiently small. There are at least two ways to check the contribution of the magnetic dipole moment. Draine, for example, compared the absorptions caused by the electric and magnetic dipole moments [5]. We prefer a direct comparison of the absolute values of the electric and magnetic dipole moments [12]. Nevertheless, both methods lead to qualitatively equivalent conclusions [12].

Draine formulated a requirement on the thickness of a skin layer of which the cell size must be smaller [5]. Note that in [5], Draine used his own definition for skin-layer thickness that gives a value greater by $4 \pi$ than the standard definition usually given in the textbooks (i.e., the distance at which the energy density of the electromagnetic wave falls to $1 / e$-its value in the space surrounding a conductor) [13]. The reason for such a modification is unclear, $\overline{a n d}$ we could only suggest that it is a typographical error. 
Such a simple explanation seems to be reasonable, taking into account the attendant text in [5]. Indeed, Draine stated: "In the event that the material is absorptive, a second necessary condition is that $d$ be small compared to the attenuation length..." However, in what follows, when discussing the thickness of the skin layer, we always use the standard definition.

It can be verified numerically that the requirement on dipole size smaller than the depth of the skin layer is more restrictive than the requirement that the contribution of the magnetic dipole moment remain small compared to the electric dipole moment (see Section 4 for details). Thus, when a conductive particle is considered, it is only enough to choose a cell size a few times smaller than the depth of the skin layer. In practice, this restriction means that within any particular cell, both the amplitude and phase of the induced electromagnetic field must be invariable. Although such a requirement seems to be intuitively clear, it has no strong theoretical background. Indeed, when deriving the Rayleigh approximation, it is only assumed that a particle is placed in a homogeneous electromagnetic field [14], that $k d \ll 1$, and also that $|m| k d \ll 1$. Note that the second condition, which restricts the complex refractive index, does allow for a significant variation of amplitude of the induced field within the particle for conductive particles; i.e., the Rayleigh-scattering criteria remain valid even if there is a significant decay in the induced field due to particle absorption. We will examine this in more detail in Section 4.

One can see that the validity criterion for the size of cells forming dielectric particles, as well as the criterion for conductive particles, arises from two subconditions: (1) satisfaction of Maxwell equations and (2) adequate reproduction of target geometry. Within the DDA, light scattering is described by a corresponding system of linear algebraic equations. Therefore, the violation of the Maxwell equations has two causes: incorrect formulation of the system of algebraic equations or its incorrect solution. In the former case, the violation could be caused by use of large constituent cells; i.e., it is possible that their light-scattering properties cannot be expressed by only the electric dipole moment, and, thus the magnetic dipole and electric quadruple moments must also be taken into account. Ignoring such a requirement will lead to violation of the Maxwell equations. Simultaneously, an incorrect solution of the system of algebraic equations might be caused, for instance, by an inappropriate choice of the iterative solver.

Inadequate reproduction of the target geometry appears due to surface roughness caused by discretization and location of the dipoles in a regular cubic lattice, which is necessary for FFT utilization. As described above, requirement (2) was found to be more restrictive than requirement (1), but the essential point of previous studies is that the results obtained with the DDA have been compared with analytical results for either a single sphere $[\underline{5}, \underline{8}]$ or two spheres in contact [8]. There is no doubt that the reproduction of scattering properties also depends on the type of particle being considered, as it is quite clear, for instance, that the spherical surface is a difficult target to reproduce using discrete cells. Therefore, we hypothesize that the criteria formulated by Draine and Flatau $[5,8,9]$ may be overstated for irregular surfaces. Indee $\bar{d}$, smoothly varying surfaces typically are not the primary consideration of DDA studies, since there are more efficient methods for such systems. For such targets, there are Mie theory [14] and the $T$-matrix method [15-19], which provide accurate and fast results. The power of the DDA lies in its ability to treat particles having an irregular structure. In such cases, surface roughness caused by a discrete representation does not cause any problems; in fact, it may even be desirable in many applications since many naturally occurring particles under consideration, such as cosmic dust particles or atmospheric dust particles, are not thought to have smoothly varying surfaces [20]. The central question we would like to attempt to answer is what sort of convergence criteria are necessary for such particles.

In what follows, we present results of numerical simulations designed to answer this question. In Section 2, we describe sample target particles and discuss the effect of orientation averaging. In Section 3 , we consider the criterion for the size of cells forming a dielectric particle, whereas Section 4 is devoted to a conductive particle. Finally, in Section $\underline{5}$, we summarize our conclusions.

\section{Description of the Target Particle and the Effect of Orientation Averaging}

As a target, we use samples of agglomerated debris particles having extremely irregular shapes. Particles of this type have been used previously to study various optical properties of irregularly shaped particles [21,22]. All computations reported in this manuscript have been made using a well-tested implementation of the DDA [10,21-24]. We also consider spheres and use the analytical Mie theory for comparison $[13,14,25]$.

The algorithm that is used to generate the agglomerated debris particles has been described, e.g., in $[21,22]$ and is not repeated here. Using this algorithm, we generate a sample particle shown in Fig. 1 (upper-right image) within a cubic matrix of $128 \bar{x}$ $128 \times 128$ cells. By removing every other plane, we can reduce the resolution by a factor of 2 . As a consequence, the resulting particle is placed in a matrix of $64 \times 64 \times 64$ elements [upper middle image of Fig. 1]. By repeating this procedure one more time, we obtain the sample particle in a matrix of $32 \times 32 \times$ 32 elements [upper-left image of Fig. 1]. The total number of cells forming the initial sample particle is 287,229 . A single reduction operation reduces the number of cells to 35,931 , whereas a double reduction operation reduces the number of cells to 4484. Nevertheless, as one can see in Fig. 1 , all three 


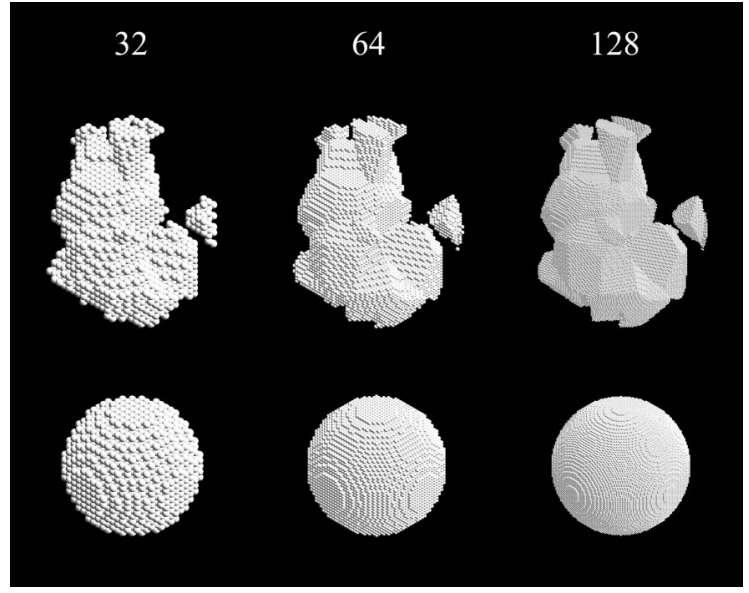

Fig. 1. Sample of agglomerated debris particles discretized in the initial matrices of different sizes (upper row) and the relevant spheres of the same volume (bottom row). The numbers represent the number of cells composing a side of the initial matrix.

representations of the sample particle reveal the same general morphology.

In addition to the irregularly shaped particle, we consider a sphere consisting of approximately the same number of cells: 285,144 cells in a $128 \times 128 \times$ 128 matrix, 35,712 cells in a $64 \times 64 \times 64$ matrix, and 4416 cells in a $32 \times 32 \times 32$ matrix. The images of these discretized spheres are shown in the bottom of Fig. 1 .

When studying the influence of large cells on light scattering, we have to take into account that they affect light scattering in two ways: through surface roughness and through mutual interference from cells located in a regular cubic lattice. We distinguish these two impacts by averaging over particle orientation. Indeed, the influence of small surface roughness on light scattering remains quite detectable even after orientation averaging [23], whereas the interference appearing in a regular grid is removed. This second influence, the result of mutual interference from cells located in a regular cubic lattice, we refer to as parasitic interference. We would like to emphasize that, according to [26], the contribution of the parasitic interference is very high; in a given orientation of a target particle, the errors of a DDA computation caused by interference coming from the regular grid can exceed all others by a significant amount. Note also that in the original study of the DDA validity criteria [8], the authors considered targets only in a fixed orientation.

In general, we consider three cases: (1) one fixed orientation of the incident beam (wave vector goes along the $z$ axis) and one plane of scattering (defined by the $z$ and $x$ axes), (2) the same orientation of the incident beam but the light-scattering properties are averaged over 360 scattering planes evenly distributed around the wave vector, which we refer to as semiaveraging, and (3) 600 random orientations of the incident beam and, in each case, the results are averaged in 360 scattering planes evenly distributed around the wave vector, which we refer to as full averaging. Thus, by increasing the degree of the orientation averaging, we can observe and isolate the effect of roughness caused by constituent cells.

\section{Dielectric Particles}

The DDA is not a new method and has been used and tested for more than 35 years. During this time, the validity criteria have been formulated $[8,9]$. We can always choose a discretization that will guarantee the accuracy of the DDA; for instance, we can further reduce the resolution parameter describing a single cell $k d|m|$ to increase the accuracy or perform DDA calculations at various resolutions and find the optical properties as $k d|m| \rightarrow 0$ [27]. In our approach, we decrease the cell size parameter to $k d|m|=0.25$, two times smaller than the most strict limitation (i.e., $0.5)$ previously discussed $[8,9]$. We consider numerical results obtained with that size parameter to be converged. Nevertheless, we confirm the reliability of the assured numerical result obtained for the agglomerated debris particle with help of Mie theory as follows: using the cells forming the irregular particle, we construct a sphere and compute its lightscattering properties with the DDA and Mie theory. We then verify the agreement between these results.

In this section, we consider light scattering by two different types of agglomerated debris particles: those with a size parameter of the circumscribing sphere $x_{\mathrm{cs}}=10$ and refractive index $m=1.6$ and those with $x_{\mathrm{cs}}=12$ and $m=1.33$. The absorption in both cases is absent. The size parameter of the circumscribing sphere is defined as $x_{\mathrm{cs}}=2 \pi r_{\mathrm{cs}} / \lambda$, where $r_{\mathrm{cs}}$ is the radius of a circumscribing sphere. We have selected these parameters so that the parameter characterizing a single cell $k d|m|$ has approximately the same value. For instance, in the largest initial matrix, i.e., that consisting of $128 \times 128 \times 128$ discrete elements, it is close to 0.25 in both cases. As a consequence, in the case of the intermediate matrix, the parameter $k d|m|$ is equal to 0.5 , whereas in the case of the smallest matrix, it is approximately 1.0.

As previously mentioned, we consider light scattering by single spheres corresponding to agglomerated debris particles, i.e., spheres consisting of approximately the same number of identical dipoles. In Figs. 2 and 3 , we present the phase curves of intensity $I$ (left panels) and degree of linear polarization $P$ (right panels) of such spheres as a function of the phase angle $\alpha$, which is supplementary to the scattering angle (i.e., $\alpha=180^{\circ}-\theta$ ), so the case of $\alpha=0^{\circ}$ corresponds to exact backscattering and $\alpha=180^{\circ}$ to exact forward scattering. Phase curves produced by a sphere corresponding to the agglomerated debris particle at $x_{\mathrm{cs}}=10$ and $m=1.6$ are shown in Fig. 2 , whereas in Fig. 3, we show phase curves produced by a sphere corresponding to the case of $x_{\mathrm{cs}}=12$ and $m=1.33$. In the former case, the size parameter of this equal-volume sphere is $x=6.379$, and in the latter case, $x=7.655$. The upper panels (a) in both figures show the results for a fixed orientation, and the lower panels (b) show results semiaveraged 

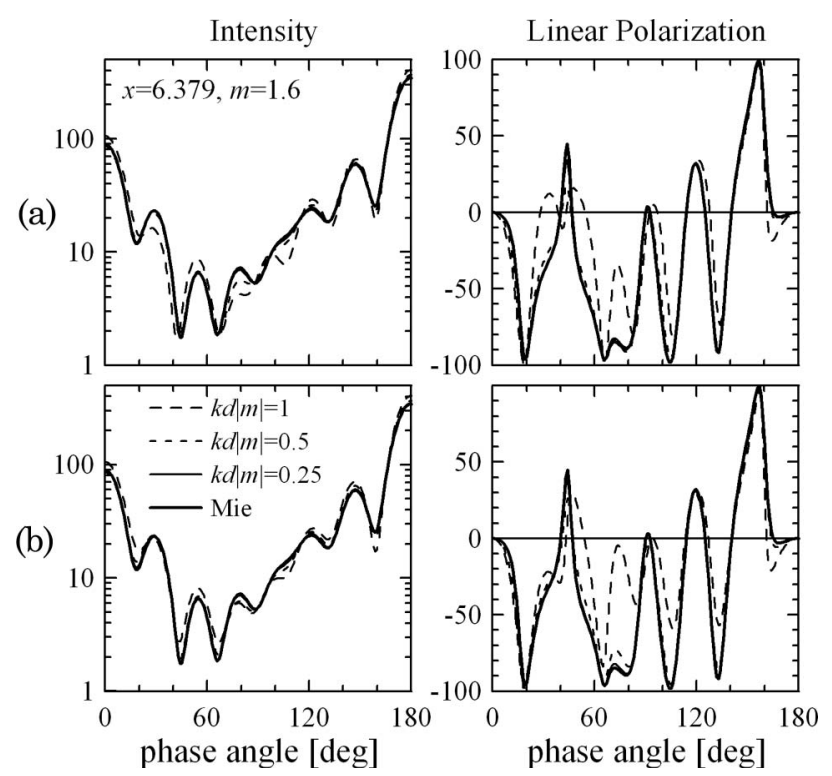

Fig. 2. Intensity (left) and degree of linear polarization (right) of a single sphere at $x=6.379$ and $m=1.6$ plotted as a function of phase angle for (a) fixed orientation and (b) semiaveraging over orientations.

over orientation. The long-dashed line shows the case of $k d|m|=1$, the short-dashed line shows the case for $k d|m|=0.5$, the thin solid line shows the case for $k d|m|=0.25$, and the thick solid line shows the exact result of Mie theory.

As one can see in the figures, the coarsest representations of a sphere $(k d|m|=1)$ reveal significant differences from the results of Mie theory. Simultaneously, the intermediate discrete representations of a sphere $(k d|m|=0.5)$ show better agreement with the exact results, although in some ranges of phase angles, the differences remain quite noticeable. The results from the finest discrete representation of a sphere $(k d|m|=0.25)$ almost coincide with those of Mie theory in Figs. 2 and 3. The fractional error of intensity traditionally has been used to quantify differences between the DDA and Mie results. As was done in [8], the error in the ith measurement is

$$
\text { Error }_{i}=\frac{\left|I_{i}^{\mathrm{DDA}}-I_{i}^{\text {exact }}\right|}{I_{i}^{\text {exact }}} \cdot 100 \%
$$

Here, $I_{i}^{\mathrm{DDA}}$ is the intensity of the scattered light calculated with the DDA and $I_{i}^{\text {exact }}$ is the intensity obtained with Mie theory. Obviously, the fractional error of intensity is a function of phase angle. In Table 1 we present three of the most commonly used values characterizing the angular dependence of the fractional error: the average error over the entire range of phase angles, the maximal error, and the standard deviations, respectively. Despite their common use, such errors are extremely misleading. The principle reason is that Mie theory predicts very sharp angular resonances in which the intensity may drop several orders of magnitude in a very small angular range. Any numerical model in which the particle is discretized will have difficulty in matching the position and magnitude of the intensity at such resonance locations, and the result is a very large error because $I_{\text {exact }}$ approaches zero. The maximal errors always occur at these locations, and the errors typically are so large at these locations that they significantly increase the average errors. From an experimental point of view, the magnitude of the intensity at these minima locations is inconsequential as the dynamic range and angular resolution of the detector system typically is not great enough to measure these values. Similar results are obtained in the polarization phase functions: if the zero-crossing points from the DDA and from Mie theory do not line up precisely, the error determined by Eq. (2) approaches infinity. Note also that the narrow oscillations in angular profiles of intensity and polarization produced by irregularly shaped particles tend to be less pronounced than in the case of single spheres, and, as a consequence, they can be more easily reproduced with the DDA.

While we present average, maximal, and standard deviation of errors for comparison, we also calculate root-mean-square errors (RMSE) and weighted rootmean-square errors $\mathrm{RMSE}_{w}$, in which the weighting function $w_{i}$ is the intensity normalized, e.g.,

$$
w_{i}=\frac{I_{i}^{\text {exact }}}{\sum_{n} I_{n}^{\text {exact }}}
$$

$$
\operatorname{RMSE}_{w}=\left[\frac{1}{1-\sum_{n}\left(w_{n}\right)^{2}} \sum_{i} w_{i}\left(\frac{I_{i}^{\mathrm{DDA}}-I_{i}^{\text {exact }}}{I_{i}^{\text {exact }}}\right)^{2}\right]^{1 / 2} .
$$

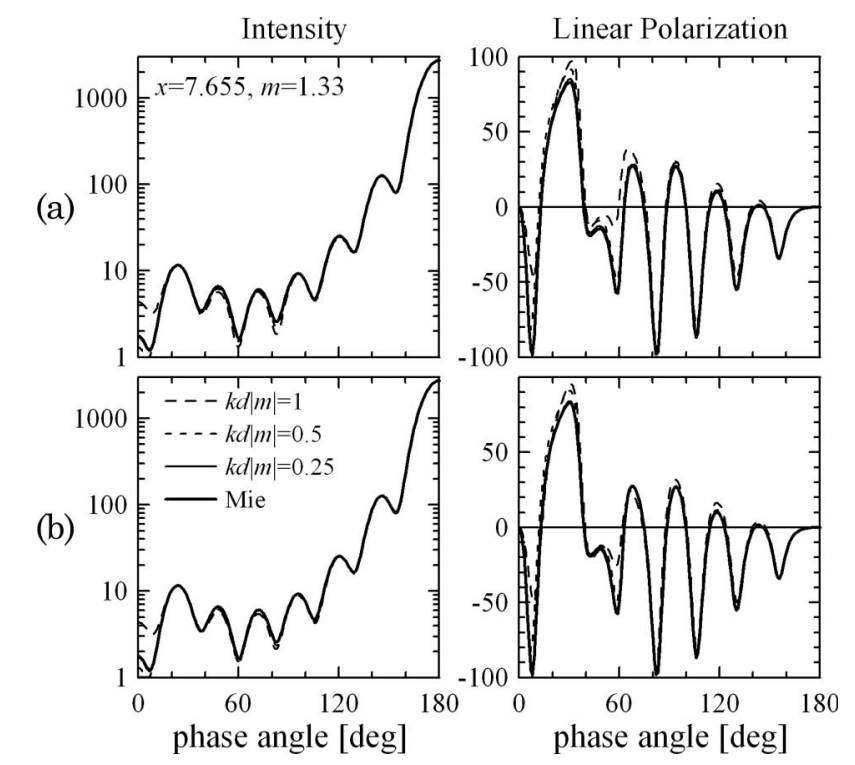

Fig. 3. Intensity (left) and degree of linear polarization (right) of a single sphere at $x=7.655$ and $m=1.33$ plotted as a function of phase angle for (a) fixed orientation and (b) semiaveraging over orientations. 
Nevertheless, the final choice of the relevant definition for numerical errors depends upon the field of use for DDA results. For example, when single scattering is being studied in respect to the radiative transfer in a dense cloud or fluffy regolith, then the weighted RMSE are quite relevant. But in the case of an extremely sparse medium, when the contribution of single scattering obviously dominates over multiple scattering, and the observations are restricted to a certain phase angle only, the fractional errors describe more adequately the accuracy of DDA computations. It is important to note the limitations of any definition of error.

Data in Table 1 quantify the conclusions made from Figs. 2 and 3. One can see that DDA simulations of light scattering by a sphere consisting of cells at $k d|m|=1$ reveal significant differences from the results of Mie theory. For instance, the maximal deviation might exceed $100 \%$, and, in fact, this happens near one of the minima in the intensity curve. Reducing the cell size by a factor of 2 (i.e., $k d|m|=$ $0.5)$ provides much higher accuracy. In this case, the average fractional error varies from $4 \%$ to $8 \%$, but its maximal deviation may be as high as $25 \%$ (the case of $x=7.655$ and $m=1.33$ ). Further decreasing the cell size to $k d|m|=0.25$ provides very good coincidence with Mie theory: the average fractional error does not exceed $2 \%$, whereas the maximal deviation of the fractional error for our sample remains less than $6 \%$.

It is interesting to note that Draine and Flatau [8] considered a single sphere at $x=7$ and $m=$ $1.33+0.01 i$. Obviously, this case is very similar to our sphere at $x=7.655$ and $m=1.33$. Therefore, the comparison of these two cases can be of interest. In the former case, the sphere has been simulated by two arrays of dipoles: 7664 and 17,904. For the case of 7664 cells, we can calculate a precise value of the parameter $k d|m|=0.76$. When comparing maximal deviations of fractional errors obtained in [8] and in the current paper, the reader may get the impression that the current DDA code is less accurate than the previous code, when, in fact, testing has shown this is not the case [10]. Indeed, at $k d|m|=0.76$, Draine and Flatau obtained a maximal deviation of $16 \%$, whereas for a similar target, our results obtained at $k d|m|=0.5$ show a maximal deviation as high as $25 \%$. The reason for this is actually an artifact of the orientation. Draine and Flatau computed light scattering at $(1,1,1)$ orientation of the incident beam [8], whereas in the present work, we consider an incident beam traveling along the $z$ axis in the $(0,0,1)$ orientation. When we repeat the previous simulation in the same conditions as [8], our code provides results having maximal deviation of less than $7 \%$. Nevertheless, if we only change the orientation of the incident beam from $(1,1,1)$ to $(0,0,1)$, the maximal deviation of the fractional errors grows up to $65 \%$. This is a demonstration of the ambiguity resulting when maximal errors are considered and resonances are a feature of the light-scattering curves. Simultaneously, full averaging over particle orientation suppresses the maximal deviation of the fractional errors to approximately $15 \%$. In general, errors in the case of a fixed orientation are higher than in the case of full orientation averaging. We would like to emphasize that the DDSCAT code developed by Draine and Flatau [9] has held up extremely well in the ensuing years. Since 1993, Draine and Flatau have undertaken many efforts in order to enhance this DDA code. As has been shown in recent comparisons, our code and DDSCAT provide comparable accuracy [10].

One method of removing the ambiguity of error results is to reduce the effect of the resonances. This can be done by weighting the error with the actual intensity, using, for instance the $\mathrm{RMSE}_{w}$ of Eq. (4). Such weightings, shown in the last column of the tables, provide more consistent results for regularly shaped particles since they are not susceptible to the large errors that can occur at the resonance locations. However, they are not necessarily ideal for all applications; for instance, because the results are intensity weighted, more emphasis is placed on data in the forward scatter region, as opposed to the backscatter region. This may or may not be desirable, depending on the application. It is a relatively trivial task to modify the weighting to suit the particular application.

Table 1. Fractional and Root-Mean-Square Errors in Intensity of Light Scattering by Dielectric Spheres Computed with the Discrete Dipole Approximation

\begin{tabular}{|c|c|c|c|c|c|c|c|}
\hline & & $k d|m|$ & $\langle$ Error $\rangle$ & $\max ($ Error $)$ & $\sigma($ Error $)$ & RMSE & $\mathrm{RMSE}_{w}$ \\
\hline \multirow{4}{*}{$x=6.379, m=1.6+0 i$} & & 0.5 & 7.50 & 23.36 & 5.21 & 9.14 & 7.03 \\
\hline & Semiaveraging & 1 & 19.83 & 68.83 & 14.16 & 24.37 & 18.95 \\
\hline & & 0.5 & 6.89 & 18.14 & 4.05 & 7.99 & 6.83 \\
\hline & & 0.25 & 2.01 & 5.57 & 1.26 & 2.37 & 1.91 \\
\hline \multirow[t]{5}{*}{$x=7.655, m=1.33+0 i$} & Fixed & 1 & 16.59 & 183.46 & 36.86 & 40.42 & 3.94 \\
\hline & & 0.25 & 0.75 & 3.88 & 0.87 & 1.14 & 0.32 \\
\hline & Semiaveraging & 1 & 14.85 & 182.28 & 36.62 & 39.52 & 3.80 \\
\hline & & 0.5 & 4.19 & 25.28 & 4.71 & 6.30 & 1.79 \\
\hline & & 0.25 & 0.58 & 3.84 & 0.78 & 0.97 & 0.30 \\
\hline
\end{tabular}


In Fig. 4 we present results for the agglomerated debris particle at $x_{\mathrm{cs}}=10$ and $m=1.6$, and in Fig. 5 we present results at $x_{\mathrm{cs}}=12$ and $m=1.33$. Unlike the figures corresponding to a sphere, we present three rows of panels. As earlier, rows (a) and (b) show the cases of one fixed orientation and semiaveraging over orientations, respectively. Additionally, row (c) shows results fully averaged over orientations. Everywhere in Figs. 4 and 5, the long-dashed line shows the case of $k d|m|=1$, the short-dashed line to $k d|m|=0.5$, and the thin solid line to $k d|m|=0.25$.

In Figs. 4 and 5, one can see that the curves corresponding to fixed orientation show the most noticeable differences between different levels of discretization of the agglomerated debris particle. Nevertheless, the distinctions are visibly smaller than those observed in the case of spheres; this especially concerns the degree of linear polarization [compare panels (a) in Figs. 2 $, \underline{4}, \underline{3}$, and $\underline{5}$ ]. Even very simple semiaveraging reduces significantly the distinctions between cases of different discretization of the nonspherical particle. Note that this type of orientation averaging has little influence on the results in the case of spheres. Full averaging over orientation almost totally suppresses distinctions between the curves of intensity corresponding to different degrees of discretization in Fig. 4 , whereas in Fig. $\underline{5}$,

(a)
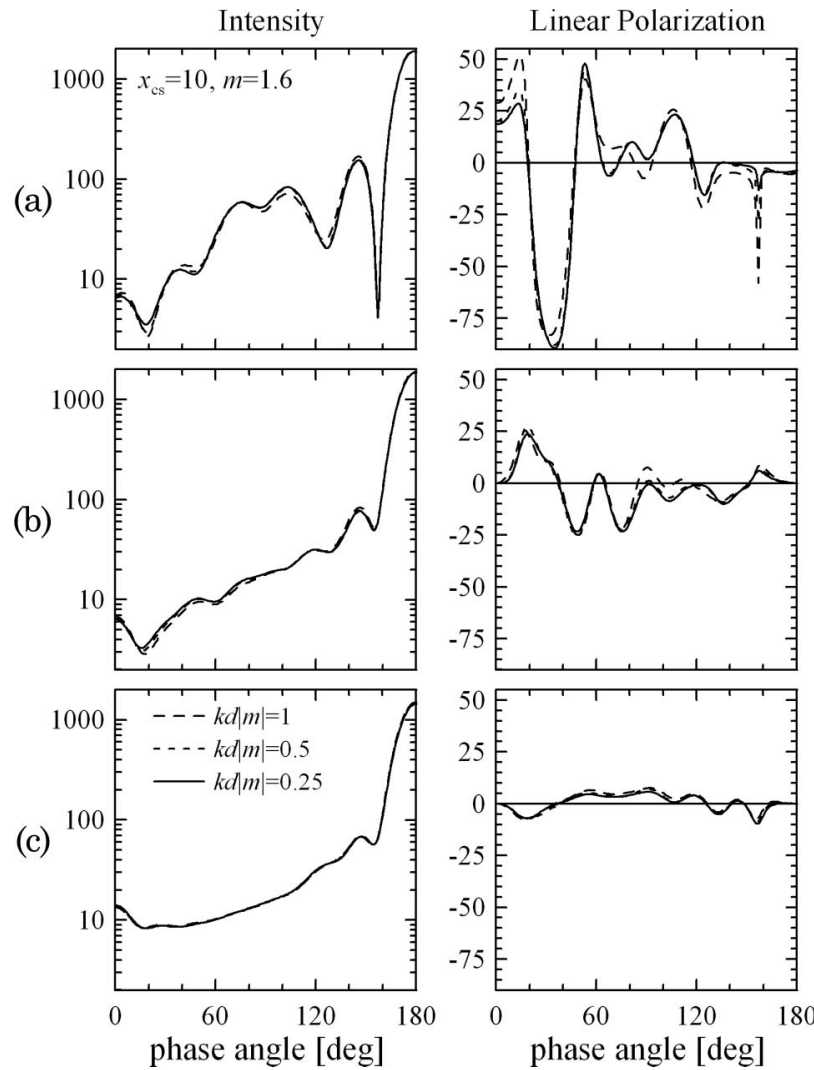

Fig. 4. Intensity (left) and degree of linear polarization (right) of an agglomerated debris particle at $x_{\mathrm{cs}}=10$ and $m=1.6$ plotted as a function of phase angle for (a) fixed orientation, (b) semiaveraging over orientations, and (c) full averaging over orientations.


Fig. 5. Intensity (left) and degree of linear polarization (right) of an agglomerated debris particle at $x_{\mathrm{cs}}=12$ and $m=1.33$ plotted as a function of phase angle for (a) fixed orientation, (b) semiaveraging over orientations, and (c) full averaging over orientations.

relatively small and nonsystematic distinctions near the backscattering are detectable.

A previous study of convergence of numerical results obtained with the DDA for spherical and cubical target shapes at different values of the parameter $k d|m|$ revealed that the convergence of a cube is faster than that of a sphere [27], thus confirming the conclusion of the current manuscript about higher accuracy of the DDA when applied to nonspherical particles. We do note that the conclusions in [27] were made based on a single orientation of the target particles, and it was demonstrated above that a change of target particle orientation may result in a large change in the amplitude of DDA errors. Therefore, the question on applicability of the conclusions in [27] to orientation averaged scatterers remains open.

In order to describe quantitatively the distinctions between the curves of intensity corresponding to different discretizations of irregular particles, we introduce the fractional errors of intensity similar to Eq. (2), but, instead of the exact value of intensity $I_{\text {exact }}$, we use the intensity obtained with the DDA at $k d|m|=0.25$. In the case of a sphere, such a value $k d|m|=0.25$ provides very good agreement between the results of the DDA and Mie theory. The parameters characterizing the errors are shown in Table 2. As follows from Table 2 , in the case of full 
Table 2. Fractional and Root-Mean-Square Errors in Intensity of Light Scattering by Dielectric Agglomerated Debris Particles Computed with the Discrete Dipole Approximation

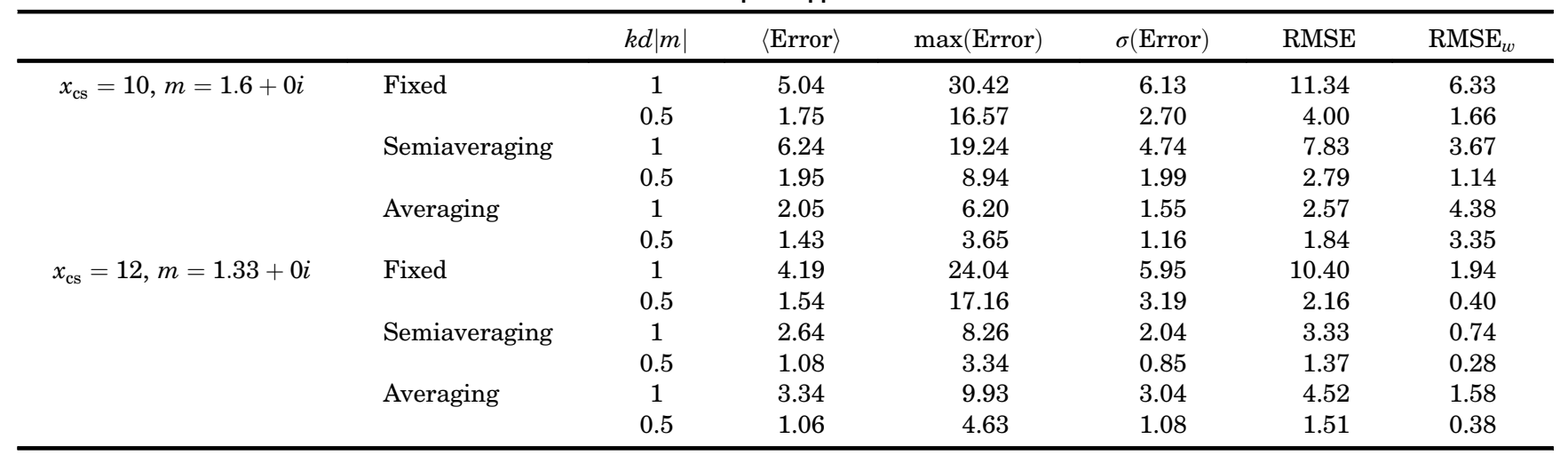

averaging over orientations at $k d|m|=1$, the average value of the fractional error of intensity is only $2 \%-4 \%$, whereas the maximal deviation of the fractional errors is $6 \%-10 \%$. Interestingly, in the case of single spheres, such accuracy can be achieved only if $k d|m|$ is significantly less than 0.5 . Thus, DDA computations of light scattering by highly irregular particles averaged over orientations can be performed accurately, even for constituent cells at $k d|m|=1$.

From Figs. 4 and 5 one can see that the polarization curves are more similar to each other due to semiaveraging over orientations than in the case of the spheres [compare with Figs. 2 and 3]. When full averaging is performed, they are nearly coincident. In other words, if some feature does exist on the polarization curve, it appears at all degrees of discretization; the value of polarization may, however, be slightly different. Because the full averaging over orientations efficiently removes parasitic interference coming from a regular grid, in panel (c) of Figs. 4 and 5, we observe the effect of discretization roughness. Indeed, as it has been shown in [23], the small-scale roughening of the thin surface layer of particles, at $x_{\mathrm{cs}}=10$ and $m=1.6+0.0005 i$, increases the depth of the negative polarization branch at small phase angles. In this manuscript, at $x_{\mathrm{cs}}=10$ and $m=1.6$, we observe the same tendency: the negative polarization depth is changed from $-7.06 \%$ $(k d|m|=0.25)$ to $-7.84 \%(k d|m|=1)$. Moreover, in [23] it was shown that the positive polarization in the intermediate range of phase angles increases due to roughening at all sets of parameters. When increasing the cell size, the same behavior is observed in all cases studied in this manuscript as well. For instance, at $x_{\mathrm{cs}}=10$ and $m=1.6$, the maximum of positive polarization is increased from $5.81 \%(k d|m|=0.25)$ to $7.51 \%(k d|m|=1)$. The discretization thus acts as roughness.

We can summarize the results reported in this section as follows. Utilization of large constituent cells $(k d|m|=1)$ raises two issues in DDA computations: surface roughness and parasitic interference coming from a regular grid. We cannot, in general, separate their contributions; however, the parasitic interference can be eliminated effectively by orientation averaging. The effect of surface roughness is not always a problem because in many applications this feature is desirable, since it replicates the roughness inherent in naturally occurring particles like dust. Nevertheless, in the case of highly irregular particles, the average contribution of the surface roughness caused by discretization does not exceed a few percent, even at $k d|m|=1$. Therefore, in numerous applications, the DDA can be applied under the condition $k d|m| \leq 1$, providing highly accurate numerical results for realistic targets.

\section{Conductive Particles}

As pointed out by Draine [5], when applying the DDA to a conductive particle, constituent cells have to be small enough to satisfy two conditions: (1) the relative contribution of the electric dipole moment must dominate significantly over the magnetic dipole moment, and (2) the cell size must be much smaller than the depth of the skin layer, providing an appropriate description of the variation of the electromagnetic field in this region. In the Introduction, we have mentioned that, in fact, the second condition is much stronger than the first, and in this section we demonstrate this numerically. For instance, we consider the largest cells used in our previous study of the influence of absorption on light scattering by agglomerated debris particles [22]. This corresponds to particles with $x_{\mathrm{cs}}=30$, which were built up in the matrix containing $128 \times 128 \times 128$ cells. At that, the parameter of the cubic constituent cells is $k d=$ 0.46875 . As a conductive material, we consider a hypothetical substance having a refractive index $m=1.5+1.3 i$. The essential advantage of the given conductor is a moderately low absolute value of its refractive index $(|m|=1.98494)$, which significantly simplifies and extends the practical utilization of the DDA.

Using Mie theory, we can estimate the electric dipole, magnetic dipole, and electric quadrupole moments of the single cell. In the theory, those are represented, correspondingly, by scattering coefficients $a_{1}, b_{1}$, and $a_{2}$, according to the notation in [25]. Because of the obvious difference between the geometrical sizes of a cube and a sphere having the same 
volume, the size parameter of the spherical equivalent of a cubic constituent cell is higher than half of the parameter $k d: x_{\text {cell }}=0.291\left(x_{\text {cell }}=k r_{\text {cell }}\right.$, where $r_{\text {cell }}$ is the radius of the spherical equivalent of the cell). According to Mie theory, at $x_{\text {cell }}=0.291$ and $m=1.5+1.3 i$, the absolute values of the scattering coefficients are as follows: $\left|a_{1}\right|=0.014013,\left|b_{1}\right|=$ 0.000178 , and $\left|a_{2}\right|=0.000061$, whereas the coefficients corresponding to the absorbing dielectric $(m=1.5+0.05 i)$ sphere of the same size are $\left|a_{1}\right|=$ $0.004856, \quad\left|b_{1}\right|=0.000058$, and $\left|a_{2}\right|=0.000023$. While all coefficients are larger for the conductive sphere, the relative contributions of the magnetic dipole and electric quadrupole moments remain approximately the same for both conductive and dielectric spheres: $\left|b_{1}\right| /\left|a_{1}\right| \approx 0.012$ and $\left|a_{2}\right| /\left|a_{1}\right| \approx 0.0045$. Therefore, the incorporation of an imaginary part of the refractive index has not increased the relative contribution of the magnetic dipole moment or the electric quadrupole moment and does not reduce the validity of the DDA.

The other criterion concerns the ratio of the cell size $d$ and the depth of the skin layer $l_{\text {skin }}$, the distance at which the energy density of the incident electromagnetic wave falls to $1 / e$ of its initial value [13]. For convenience, instead of pure values of $d$ and $l_{\text {skin }}$, we consider their products with wavenumber $k$ : $k d$ and $k l_{\text {skin }}=0.5 / \operatorname{Im}(m)$. In the case of $k d=$ 0.46875 and $m=1.5+1.3 i$, the parameter $k l_{\text {skin }}$ is equal to only 0.38462 . Thus the depth of the skin layer is actually smaller than the size of a single cell, whereas the skin layer must be at least a few times larger than a cell in order to reproduce adequately the attenuation of the electromagnetic field. Therefore, the requirement on the depth of the skin layer is much stronger than that on the relatively small contribution of the magnetic dipole moment.

Except when the particle is on resonance or is extremely conductive, the restriction of amplitude invariance of the induced field within the volume of a small particle does not alter the resulting light scattering from Rayleigh theory [28]. In such instances of extremely large refractive in $\overline{d e x} m$, the induced field within the particle is not constant, yet the resulting scattering properties can still be predicted using Rayleigh theory, except when the particle is on resonance. This result demonstrates that an induced field that is constant over the dipole is not a necessary condition for Rayleigh theory to hold. Below we present numerical results showing how this is relevant to DDA calculations.

For this purpose, we compute the distribution of energy of the induced field in a volume of a conductive particle at different levels of discretization using the DDA. The numerical results agree with the wellknown theoretical prediction, which is as follows: the electromagnetic field does not penetrate into a conductive particle and the induced electromagnetic field exists only in a very shallow near-surface layer. Two targets have been considered: a single sphere at $x=4.795$ and an agglomerated debris particle at $x_{\mathrm{cs}}=7.5$. Both particles consist of approximately the same volume of conductive material at $m=1.5+$ $1.3 i$, and their constituent cells are identical. In the smallest initial matrix with dimensions of 32 elements on a side, the parameter $k d|m|=0.93$ (i.e., $k d=0.46875$ ); thus, the skin depth is smaller than the size of a cell. We make corresponding simulations for matrices that are twice and four times as large, i.e., the cells are two and four times smaller, and $k d|m|=0.47$ and 0.23 , respectively; thus, the skin layers are approximately 1.5 and 3 times larger than cells. Therefore, in the last case, both validity criteria formulated by Draine [5] are formally satisfied.

The images of the distribution of the induced field energy over the volume of the targets are shown in Fig. 6. The distribution has been normalized, so the energy varies from 0 to 1 through the array of dipoles. Panel (a) shows results for a sphere, whereas panel (b) shows results for the agglomerated debris particle. In both cases, the direction of the incident wave propagation coincides with the $z$ axis, which is shown by a white line in the upper-left corner of

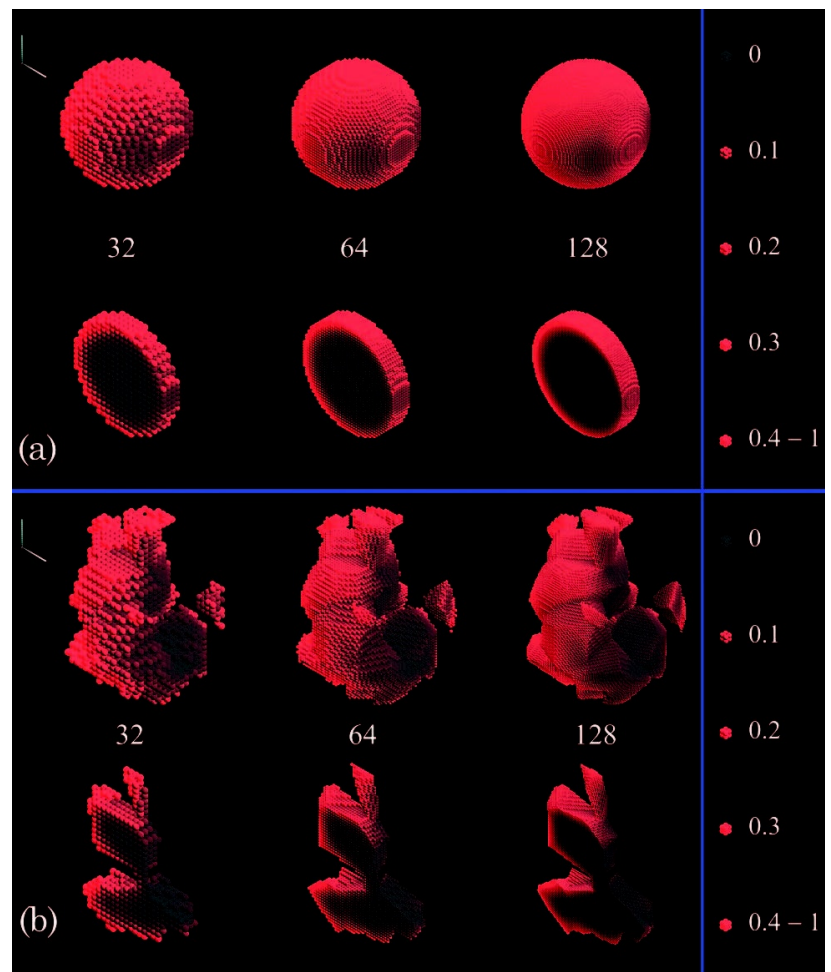

Fig. 6. (a) Distribution of energy of the induced field in spheres and (b) agglomerated debris particles consisting of different numbers of cells. The refractive index is $m=1.5+1.3 i$. The size parameter of the sphere is $x=4.795$, whereas the size parameter of the circumscribing sphere of the agglomerated debris particle is $x_{\mathrm{cs}}=7.5$. In each panel, the directions of propagation and polarization of the incident beam are shown by white and blue lines in the upper-left corner, respectively. The upper row shows whole particles, whereas the bottom row shows their cross sections. When the particle is allocated in the matrix with a side composed of 32 cells, the parameter of a single cell $k d|m|=0.93 ; 64$ cells corresponds to $k d|m|=0.47$; and 128 cells corresponds to $k d|m|=0.23$. 
each panel. The incident wave is linearly polarized, and the direction of polarization coincides with the $x$ axis and is shown by the blue line. Each target particle appears at three different degrees of discretization; sizes of the corresponding initial matrices are indicated below each particle. At the bottom of each panel, we also show the distribution of the induced field energy through cross sections of the targets. Note that the color scale in Fig. 6 is not linear: the bright red color shows dipoles having energy of the induced field varying from 0.1 to 1 .

As one can see from Fig. 6 , in all degrees of discretization, the DDA provides the same distribution of energy of the induced field, which in turn agrees qualitatively with that predicted for conductors. The details appearing at the highest resolution discretization also can be seen at the lowest resolution. The averaging of the amplitude of the induced electromagnetic fields over a cell size does not appear to have affected the distribution of energy over the particle; however, it could have an influence on the angular profiles of intensity and degree of linear polarization, as well as on other light-scattering parameters.

In Fig. 7 we present curves of intensity (left panels) and degree of linear polarization (right panels) of the sphere at $x=4.795$ and $m=1.5+1.3 i$. The organization of the figure is the same as that of Figs. 2 and 3, namely, the upper panel corresponds to the fixed orientation and the bottom panel corresponds to the semiaveraging over orientations. When the number of cells forming the sphere increases, the angular profiles of intensity and degree of linear polarization approach those of the Mie-theory results. Nevertheless, even the most accurate result obtained with the DDA (i.e., in the matrix of $128 \times 128 \times 128$ cells) is still visibly different from that of Mie theory,
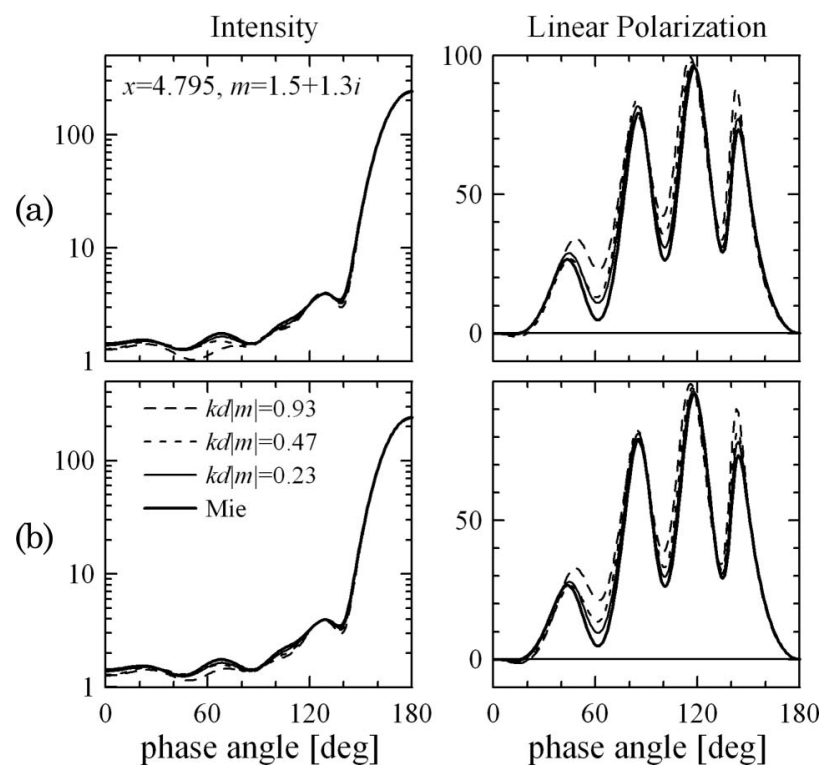

Fig. 7. Intensity (left) and degree of linear polarization (right) of a single sphere at $x=4.795$ and $m=1.5+1.3 i$ plotted as a function of phase angle for (a) fixed orientation and (b) semiaveraging over orientations. especially the curve of the linear polarization degree. For the dielectric particles studied in the previous section at a similar value of parameter $k d|m|$, the differences were much smaller. The decreasing accuracy for conductive particles can be explained as follows. In the case of a conductive particle, the induced field appears only near the surface layer. Indeed, as one can see from Fig. 6 on the illuminated side of the sphere, the incident field penetrates into the volume much deeper than on the shadow side, whereas in between, the depth of the induced layer is being gradually changed. In the case of a perfect sphere, that layer has ideally smooth boundaries and its light scattering can be described precisely with Mie theory, whereas with the DDA, both boundaries of the induced layer have discrete structure, and, as a consequence, it is more difficult to reproduce the gradual change of the layer depth accurately, even using a large number of cells. Therefore, we can conclude that the effect of the discretization has an additional negative impact on the accuracy of DDA computations for conductive particles.

One other consideration is the surface wave. For conductive particles, the surface wave is the dominant component, inducing dipole moments on the backside of particles since the transmitted component is rapidly truncated. Dipoles do not efficiently bend the surface wave around curved surfaces. For instance, one can see that the amplitude of the surface wave on the rear of the particles is reduced as the resolution is decreased from the panels of Fig. 6 . In panel (b), the highly irregular structure with surface elements of high curvature efficiently destroys the surface wave. Indeed, the induced layer is almost absent from the nonilluminated side of the agglomerated debris particle, in sharp contrast to the illuminated side. This depletion of the surface layer, which is a dominant component of conductive surfaces, is a source of significant errors in DDA computations.

Table 3 shows values characterizing the angular dependence of the fractional errors (2) and RMSE for three levels of discretization of a sphere with $x=$ 4.795 and $m=1.5+1.3 i$. Comparing the data from Tables 1 and 3 , we could conclude that the results obtaine $\bar{d}$ for the case of a conductive sphere is more accurate than those for a dielectric sphere. However, this conclusion is superficial. Indeed, as it was earlier elucidated, the DDA result obtained at one orientation of a particle strongly depends on the interference coming from the regular grid, and for a spherical particle, on the exact positions of the angular resonances. Depending on the orientation of the incident beam, that contribution may increase or decrease the accuracy randomly, whereas the difference between the best and worst accuracy can be as high as an order of magnitude. For instance, if we change the orientation of the incident beam from the initial $(0,0,1)$ to $(1,1,1)$, the values 〈Error $\rangle, \max ($ Error), and $\sigma($ Error $)$ characterizing the fractional errors in the upper row of Table 3 change to $16.20,28.26$, and 8.76 , respectively. One can see that the new value 
Table 3. Fractional and Root-Mean-Square Errors in Intensity of Light Scattering by a Conductive Sphere Computed with the Discrete Dipole Approximation

\begin{tabular}{|c|c|c|c|c|c|c|c|}
\hline & & $k d|m|$ & $\langle$ Error $\rangle$ & $\max ($ Error $)$ & $\sigma($ Error $)$ & RMSE & $\mathrm{RMSE}_{w}$ \\
\hline \multirow{4}{*}{$x=4.795, m=1.5+1.3 i$} & & 0.47 & 5.27 & 14.70 & 4.20 & 6.74 & 2.24 \\
\hline & Semiaveraging & 0.93 & 6.30 & 20.69 & 6.32 & 10.20 & 3.77 \\
\hline & & 0.47 & 3.72 & 10.55 & 3.41 & 6.12 & 2.05 \\
\hline & & 0.23 & 1.97 & 5.73 & 1.86 & 3.61 & 1.18 \\
\hline
\end{tabular}

of the average fractional errors of intensity has been increased by a factor of almost 2 and is now comparable to the value in the case of the dielectric sphere at $x=7.655$ and $m=1.33$. Simultaneously, the change in the percent deviation of the RMSE is slightly less, going from $12.75 \%$ to $18.41 \%$. As expected, the intensity-weighted $\mathrm{RMSE}_{w}$ changes the least, from $4.26 \%$ to $5.28 \%$. This tendency has been observed throughout the data analysis, and also holds for irregularly shaped particles. Therefore, we can conclude that the weighted RMSE remain the most stable to random variations caused by different orientations. Because of its stability, the intensity-weighted $\mathrm{RMSE}_{w}$ seems to provide the most reliable and efficient way of estimating the merit of such calculations. Note also that in the case of the angular profile of intensity, semiaveraging over orientation improves significantly the accuracy of the DDA results, whereas in the case of degree of linear polarization, this type of averaging has a rather small effect. Such behavior is different from what was observed for dielectric spheres, in which case the semiaveraging over orientation had a weak effect on both the intensity and the polarization.

In Fig. 8, we show results for the agglomerated debris particle at $x_{\mathrm{cs}}=7.5$ and $m=1.5+1.3 i$. As previously, in the case of dielectric agglomerated debris particle, the figure consists of three rows of panels. The (a) panels show results for fixed orientation, the (b) panels show results semiaveraged over orientation, and the (c) panels show results totally averaged over orientation. In all three cases, the dispersion of curves of the intensity, and the degree of linear polarization corresponding to different discretizations, is much smaller than for a sphere. At that, the increasing degree of orientation averaging reduces the difference between the polarization curves obtained at different levels of discretization, whereas it has no significant influence on the intensity curves.

One can see that, through all the panels in Fig. 8 , the difference between the DDA results calculated for initial matrices consisting of $64 \times 64 \times 64$ and of $128 \times 128 \times 128$ cells remains appreciably small. Thus, we can extend again the definition of the fractional errors in intensity [see Eq. (2)] for the case of the agglomerated debris particles. The values characterizing the angular profile of fractional errors in intensity and RMSE are summarized in Table 4.

The case of full averaging over orientations is of special interest because the interference effects caused by the regular grid are eliminated; therefore, we can isolate the effect of averaging of the induced field amplitude over cell volume. Obviously, the effect of such averaging becomes most apparent at the largest value of $k d|m|$ (i.e., 0.93). Nevertheless, as one can see in panel (c) of Fig. 8 , the difference between curves corresponding to different cell sizes remains only quantitative; i.e., the increase of cell size does not lead to the appearance of new features on curves of intensity and degree of linear polarization. As follows from Table $\underline{4}$, the average fractional errors in intensity are approximately $8 \%$, whereas their maximal deviation is as high as $13 \%$. Although these values are a few times larger than in the case of

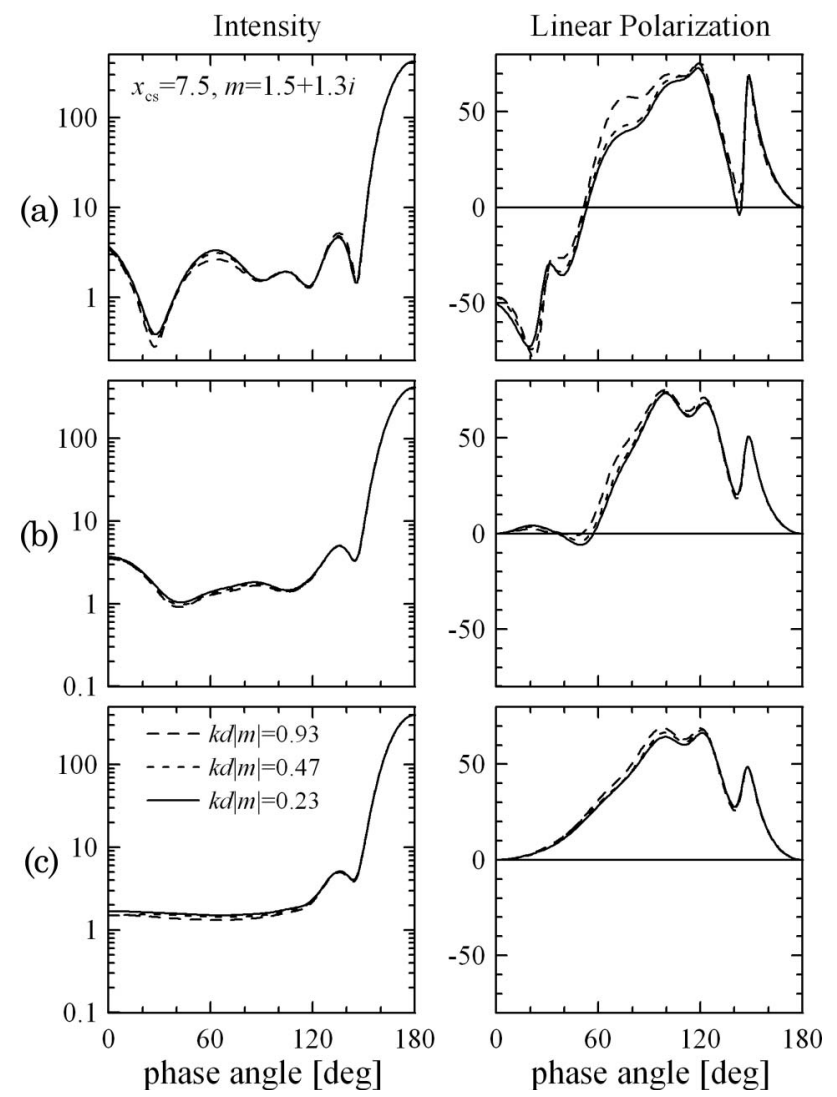

Fig. 8. Intensity (left) and degree of linear polarization (right) of an agglomerated debris particle at $x_{\mathrm{cs}}=7.5$ and $m=1.5+1.3 i$ plotted as a function of phase angle for (a) fixed orientation, (b) semiaveraging over orientations, and (c) full averaging over orientations. 
Table 4. Fractional and Root-Mean-Square Errors in Intensity of Light Scattering by a Conductive Agglomerated Debris Particle Computed with the Discrete Dipole Approximation

\begin{tabular}{|c|c|c|c|c|c|c|c|}
\hline & & $k d|m|$ & $\langle$ Error $\rangle$ & $\max ($ Error $)$ & $\sigma($ Error $)$ & RMSE & $\mathrm{RMSE}_{w}$ \\
\hline \multirow[t]{6}{*}{$x_{\mathrm{cs}}=7.5, m=1.5+1.3 i$} & Fixed & 0.93 & 4.43 & 28.76 & 6.22 & 8.37 & 2.03 \\
\hline & & 0.47 & 1.89 & 9.93 & 2.31 & 3.77 & 0.88 \\
\hline & Semiaveraging & 0.93 & 5.51 & 13.14 & 3.99 & 6.80 & 1.53 \\
\hline & & 0.47 & 3.02 & 6.43 & 2.10 & 3.68 & 0.82 \\
\hline & Averaging & 0.93 & 8.05 & 13.08 & 4.58 & 9.26 & 2.12 \\
\hline & & 0.47 & 3.59 & 10.99 & 2.73 & 4.51 & 1.02 \\
\hline
\end{tabular}

dielectric particles, nevertheless they remain at quite acceptable levels for many applications.

It is interesting to note that the deviation of the intensity curve corresponding to the case of $k d|m|=$ 0.93 from that of $k d|m|=0.23$ appears mostly in the range of intermediate and small phase angles through systematically understating values of intensity for $k d|m|=0.93$. Simultaneously, the linear polarization degree is increased while the cell size increases. Both facts are consistent with the conclusions of our previous study of the surface roughness impact on light scattering [23]. Therefore, the reaction of the intensity and polarization profiles on increasing cell size is analogous to what is observed when surface roughness is introduced onto a particle.

We can summarize the results reported in this section as follows: strictly speaking, an application of the DDA to conductive particles is specified by only one necessary condition; namely, the contribution of the electric dipole moment must be significantly greater than the magnetic dipole and electric quadrupole moments. If that condition is satisfied, the numerical results obtained using the DDA satisfy the Maxwell equations. An insufficiently accurate simulation of the particle generally does not result in problems with the satisfaction of Maxwell equations through the numerical results; nevertheless, it can alter significantly the light-scattering properties through discretization errors. In the case of angular profiles of intensity and degree of linear polarization, the influence is similar to that produced by surface roughness. Therefore, insufficiently accurate simulation of the skin layer can be interpreted as an additional small-scale roughening of a target particle.

\section{Conclusion}

In this study we have investigated the validity criteria for the DDA in its application to dielectric and conductive agglomerated debris particles. We found that the widely used criteria established by Draine and Flatau are overly restrictive with regard to satisfying the Maxwell equations. The reason for this deviance is as follows. When examining the DDA, Draine and Flatau used a perfect sphere as a test particle. Such a particle requires a large number of discrete cells in order to reproduce the surface precisely, and the smooth regular surfaces encourage the formation of standing waves within the particle that result in large-amplitude angular structures in the phase function. Such standing waves are difficult to pro- duce in a discretized system, and the resulting errors due to the large-amplitude swings are dominated by the minima, that in many respects, are inconsequential when considering real particle systems. The criteria introduced by Draine and Flatau are not relevant to the satisfaction of Maxwell equations, but to the representation of this ideal geometry. Fortunately, a sphere or other particles having a perfectly smooth surface are not the primary goal for the DDA. The most important advantage of the DDA is that it is applicable to particles of rough, irregular shape and internal structure. Therefore, this method is especially suitable for highly irregular particles, such as dust, when other approaches are not available. For such particles, surface roughness is a characteristic inherent in the discretization process of the DDA that is desirable, making a model particle more realistic. The effect of the discretization roughness on the light-scattering curves has the same qualitative effect as incorporating real roughness on simulated particles. We have shown that when light-scattering properties are averaged over particle orientation, the average impact of the surface roughness caused by large cells (i.e., at $k d|m|=1$ ) on the angular profile of intensity is only a few percent. In the case of conductive particles, large cells may lead to a situation in which the cell size is comparable to the depth of the skin layer. Although this means a considerable averaging of the induced field over cell volumes, this does result in a violation of the Maxwell equations. Again, the effect of using larger cells for conductive particles is consistent with adding surface roughness. Simultaneously, the orientation averaging efficiently increases the accuracy of DDA results.

We are grateful to Maxim Yurkin for his comments on this paper. This work was partially supported by Grants-in-Aid for Scientific Research (A and B) from the Japan Society for the Promotion of Science, Global Centers of Excellence (COE) Program "Foundation of International Center for Planetary Science" from the Ministry of Education, Culture, Sports, Science and Technology of Japan, and the Academy of Finland (contracts 1127461 and 125180).

\section{References}

1. E. M. Purcell and C. R. Pennypacker, "Scattering and absorption of light by nonspherical dielectric grains," Astrophys. J. 186, 705-714 (1973).

2. P. R. Shapiro, "Interstellar polarization: magnetite dust," Astrophys. J. 201, 151-164 (1975). 
3. S. D. Druger, M. Kerker, D.-S. Wang, and D. D. Cooke, "Light scattering by inhomogeneous particles," Appl. Opt. 18, 38883889 (1979).

4. Y. L. Yung, "Variational principle for scattering of light by dielectric particles," Appl. Opt. 17, 3707-3709 (1978).

5. B. Draine, "The discrete-dipole approximation and its application to the interstellar graphite grains," Astrophys. J. 333, 848-872 (1988).

6. J. J. Goodman, B. T. Draine, and P. J. Flatau, "Application of fast-Fourier-transform techniques to the discrete-dipole approximation," Opt. Lett. 16, 1198-1200 (1991).

7. B. T. Draine and J. J. Goodman, "Beyond Clausius-Mossotti: wave propagation on a polarizable point lattice and the discrete dipole approximation," Astrophys. J. 405, 685-697 (1993).

8. B. T. Draine and P. J. Flatau, "The discrete dipole approximation for scattering calculations," J. Opt. Soc. Am. A 11, 14911499 (1994).

9. B. T. Draine and P. J. Flatau, "User guide for the discrete dipole approximation code DDSCAT 6.1" (2004), available at http://arxiv.org/abs/astro-ph/0409262v2.

10. A. Penttilä, E. Zubko, K. Lumme, K. Muinonen, M. A. Yurkin, B. Draine, J. Rahola, A. G. Hoekstra, and Y. Shkuratov, "Comparison between discrete dipole implementations and exact techniques,” J. Quant. Spectr. Rad. Trans. 106, 417-436 (2007).

11. M. A. Yurkin, V. P. Maltsev, and A. G. Hoekstra, "The discrete dipole approximation for simulation of light scattering by particles much larger than the wavelength," J. Quant. Spectr. Rad. Trans. 106, 546-557 (2007).

12. E. Zubko, D. Petrov, Y. G. Shkuratov, H. Okamoto, K. Muinonen, H. Kimura, T. Yamamoto, and G. Videen, "Applicability of discrete-dipole approximation to conductive particles," in Proceedings of the Eleventh Conference on Electromagnetic and Light Scattering by Nonspherical Particles: Theory, Measurements, and Applications (University of Hertfordshire, 2008), pp. 117-120.

13. M. Born and E. Wolf, Principles of Optics, 7th ed. (Cambridge U. Press, 1999).

14. H. C. van de Hulst, Light Scattering by Small Particles (Dover, 1981).

15. P. C. Waterman, "Symmetry, unitarity, and geometry in electromagnetic scattering," Phys. Rev. D 3, 825-839 (1971).
16. M. I. Mishchenko, L. D. Travis, and A. A. Lacis, Scattering, Absorption, and Emission of Light by Small Particles (Cambridge U. Press, 2002).

17. D. Petrov, E. Synelnyk, Y. Shkuratov, and G. Videen, "The $T$ matrix technique for calculations of scattering properties of ensembles of randomly oriented particles with different size," J. Quant. Spectr. Rad. Trans. 102, 85-110 (2006).

18. D. Petrov, Y. Shkuratov, and G. Videen, "Analytical lightscattering solution for Chebyshev particles," J. Opt. Soc. Am. A 24, 1103-1119 (2007).

19. D. Petrov, Y. Shkuratov, and G. Videen, "Influence of corrugation on light-scattering properties of capsule and finitecylinder particles: Analytic solution using Sh-matrices," J. Quant. Spectr. Rad. Trans. 109, 650-669 (2008).

20. T. Nousiainen, "Optical modeling of mineral dust particles: a review," J. Quant. Spectr. Rad. Trans. 110, 1261-1279 (2009).

21. E. Zubko, Yu. Shkuratov, N. N. Kiselev, and G. Videen, "DDA simulations of light scattering by small irregular particles with various structure,” J. Quant. Spectr. Rad. Trans. 101, 416-434 (2006).

22. E.Zubko,H.Kimura, Yu.Shkuratov,K.Muinonen,T.Yamamoto, H. Okamoto, and G. Videen, "Effect of absorption on light scattering by agglomerated debris particles," J. Quant. Spectr. Rad. Trans. 110, 1741-1749 (2009).

23. E. Zubko, K. Muinonen, Yu. Shkuratov, G. Videen, and T. Nousiainen, "Scattering of light by roughened Gaussian random particles," J. Quant. Spectr. Rad. Trans. 106, 604-615 (2007).

24. E. Zubko, Yu. Shkuratov, M. Hart, J. Eversole, and G. Videen, "Backscattering and negative polarization of agglomerate particles," Opt. Lett. 28, 1504-1506 (2003).

25. C. F. Bohren and D. R. Huffman, Absorption and Scattering of Light by Small Particles (Wiley, 1983).

26. H. Okamoto, "Information content of the $95-\mathrm{GHz}$ cloud radar signals: theoretical assessment of effects of nonsphericity and error evaluation of the discrete dipole approximation," J. Geophys. Res. 107, 4628 (2002).

27. M. A. Yurkin, V. P. Maltsev, and A. G. Hoekstra, "Convergence of the discrete dipole approximation. I. theoretical analysis," J. Opt. Soc. Am. A 23, 2578-2591 (2006).

28. G. Videen and W. S. Bickel, "Light scattering resonances in small spheres," Phys. Rev. A 45, 6008-6012 (1992). 